\title{
Comparison of the microstructure of boron-based diffusion coatings obtained from different pastes
}

\author{
A.M. Guriev, S.G. Ivanov ${ }^{\dagger}$, M.A. Guriev \\ †serg225582@mail.ru
}

Altay State Technical University after I.I. Polzunov, 46 Lenina St., 656038, Barnaul, Russia

In this paper we studied the microstructure of diffusional coatings based on boron and titanium, obtained from different pastes. Using energy-dispersive analysis, the elemental composition of the diffusion coatings was determined. The influence of the elemental composition and microstructure on the operational life of products, hardened in accordance with the proposed technology, in the conditions of abrasive wear in sulfuric acid solution was investigated.

Keywords: boriding, hardening, diffusion.

\section{Introduction}

The majority of machine tools, parts and working elements operate in the conditions of surface wear. It follows from this that it is the structure and properties of the surface layers that will determine, to a great extent, the operational life of the product on the whole. Among the existing methods of surface hardening, the most widespread are the different methods of coating application: welding deposition, spraying, mechanical surface treatment (e.g., diamond smoothing), thermal treatment, thermochemical treatment.

From the above-mentioned methods of surface hardening, the most advantageous is the method of thermochemical treatment (TCT). This method normally does not require any specialized equipment, which is often expensive, and the TCT process can be easily integrated in the existing chain of processing operations, and is fully compatible with thermal treatment. Among the promising methods of thermochemical treatment are such methods as chromizing, titanizing, boriding, aluminizing and the combined processes of saturation with several elements (chrome aluminizing, boron chromizing, boron titanizing, titanium aluminizing, etc.).

This work is devoted to the study of the process of simultaneous saturation of carbon steel 45 with boron, chromium and titanium. In papers on this topic, published earlier, there is information that the process of simultaneous boron-titanizing proceeds ambigously: often the process of single-component boriding takes place, but the boride layer in that case has a thickness that is not sufficiently large to be favorable for increasing the operational life of the hardened product under complex conditions. Therefore, the recommended application area for products hardened by boron-titanizing is the operation predominantly in aggressive environments containing alkaline solutions and mineral acid solutions. The papers [1-3] contain information that the boron diffusion process is strongly intensified when chromium compounds are added to the saturating medium, but even in this case, the processes of simultaneous saturation with boron and titanium run very slowly, and predominantly either the boriding process or the titanizing process is realized. Simultaneous saturation with boron, chromium and titanium was achieved only for the high-alloyed steel Kh12M.

\section{Experimental procedure}

Experiments on simultaneous saturation of carbon steel 45 with boron, chromium and titanium were conducted. The saturation process was carried out in a chamber furnace of the SNOL type, equipped with a «Thermodat 16E3» PID controller that allows to control temperature with an accuracy of $\pm 1^{\circ} \mathrm{C}$ in the range of temperatures from 20 to $1350^{\circ} \mathrm{C}$. The saturation was implemented from pastes based on boron carbide [4-7], to which chromium diboride and ferrotitanium were added in the case of one-layer paste application, and chromium dibordibe was added in the case of two-layer application. In the case of two-layer application, ferrotitanium was used as an independent layer with a thickness of $0.1-0.2 \mathrm{~mm}$, applied immediately on the surface of the hardenable steel, and on top of it another layer was applied containing boron carbide and chromium diboride. In the case of one-layer application, ferrotitanium was added to a paste based on boron carbide and chromium dibordide, in the amount of $15 \mathrm{wt} . \%$ of the paste quantity. The total thickness of applied paste in both cases was $4-5$ $\mathrm{mm}[5,6]$. After application of the paste, the samples were subjected to drying until the formation of a hard crust, thereafter they were placed into a furnace, heated in advance to $930^{\circ} \mathrm{C}$, and held at this temperature for 1.5 hours. After the holding time was over, the samples were removed from the furnace, air-cooled to room temperature, thereafter the paste residue was removed and the samples were washed in soapy water. 
The samples for the study were prepared using a «MICRACUT - 201» high-precision cutting machine. On this machine, plates with a thickness of $0.5 \mathrm{~mm}$ were cut and then pressed in phenolic resin on a "METAPRESS» metallographic press and mechanically polished on a «DIGIPREP» automatic polishing machine. The obtained metallographic sections were etched with a $4 \%$ solution of nitric acid in ethanol to identify the general microstructure, or with a $5 \%$ iodine solution in ethanol to identify the phase composition of the diffusion boride layer: here the high-boron phase gets coloured in a darker colour, and the low-boron phase remains bright. Electron-microscopy microstructural studies were performed on non-etched sections, using the BSE detector that allows to obtain images with information about the composition variations on the basis of the average atomic number contrast.

The preliminary microstructural studies were performed on a Carl Zeiss Axio Observer Z1m optical inverted microscope. The preliminary investigations of the elemental composition were conducted using a X-MET 7500 X-ray fluorescence express-analyzer. To perform the main studies, a TESKAN MIRA 3 LMU electron microscope was used, which was equipped with an energy-dispersive analysis system based on the X-MAX Pro sensor and the Aztec Advanced analytical system.

\section{Results and discussion}

Figures 1 and 2, respectively, display the optical and electron images of cross sections of the diffusion coatings produced by simultaneous saturation with boron, chromium and titanium from one-layer and two-layer paste, correspondingly.

As can be seen from the given images, the microstructure of the diffusion coatings depends on the saturation method and is drastically different in terms of both morphology and the elemental composition. The boron content was measured with calibration against a lanthanum hexaboride LaB6 reference specimen, the measurement error in this case does not exceed 3\%. For light elements in various types

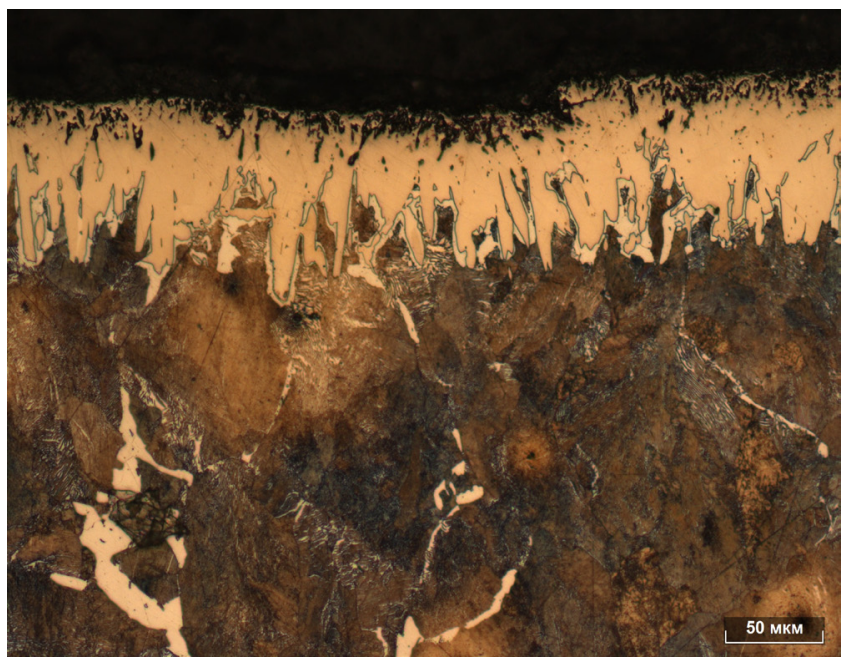

of compounds (in which the light element is an electron donor or acceptor), the electron density of states is shifted considerably, and as a result, the error in their measurement by energy-dispersive analysis grows.

In the case of simultaneous saturation of steel 45 from a one-layer paste containing boron, chromium and titanium, the process of predominant boriding takes place, and all other elements except iron are present in the quantities normally contained in this steel grade, as testified by the data from energy-dispersive analysis listed in Table 1.

The diffusion layer, in the case of saturation from a onelayer paste, has a needle-like appearance typical for boride layers. In the case of saturation from a two-layer paste, a smooth layer is formed, but the boron content in it exceeds the boron content in the needle-like layer, according to the energy-dispersive analysis data $[8,9]$.

Besides, in the image showing the diffusion coating obtained from a two-layer paste, cracks can be observed, which may indicate either high brittleness of this coating, or a high level of stresses emerging in the process of saturation and subsequent cooling and leading to cracking of the coating.

The hypothesis of a high level of stresses emerging in the process of saturation is supported by the image of the diffusion coating surface presented in Fig.3. It can be seen that on the surface of the diffusion coating there are ruptures in the form of surface cracks. The most probable cause for appearance of such cracks is the difference in the thermal expansion coefficients for the coating and the base metal. As a result of a considerable «shrinkage» of the coating and the stresses, higher than the yield stress of the diffusion layer, emerging due to that during cooling of the hardened product, cracks appear there. However, as can be seen from Fig.2b, these cracks are not through-thickness cracks that pass through the whole diffusion layer to the base metal (the core). Owing to that, as well as to the fact that the coating in this case has a more complex chemical composition, its durability in the conditions of abrasive wear in the sulfuric acid solution is, on average, 1.8-2.6 times higher, as compared with that of the coatings obtained from a one-layer paste where titanium was a component of the paste.

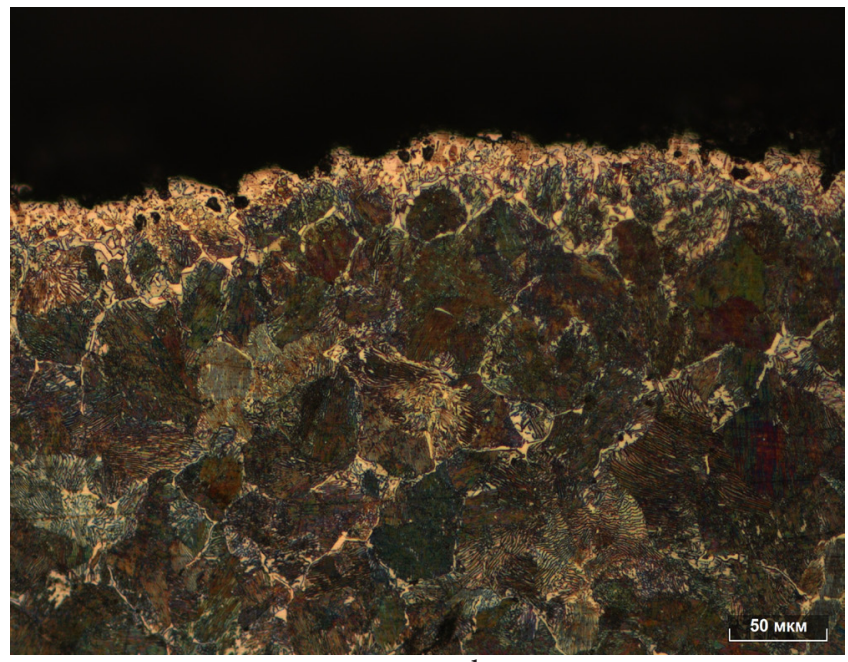

$\mathrm{b}$

Fig. 1. Optical images of the microstructure of diffusion coatings on steel 45 produced by complex saturation with boron, chromium and titanium from pastes: (a) saturation from a one-layer paste; (b) saturation from a two-layer paste. 


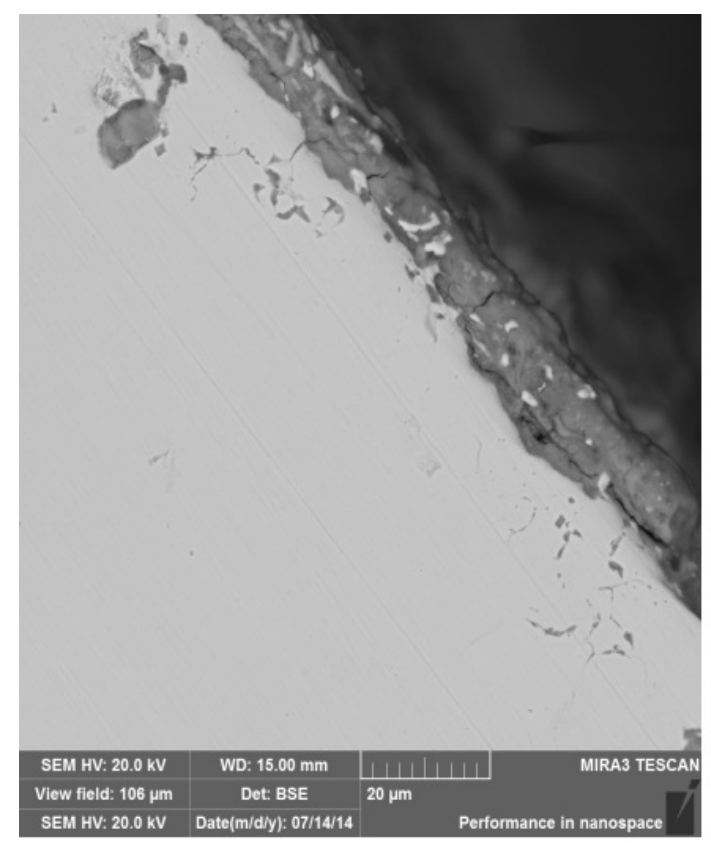

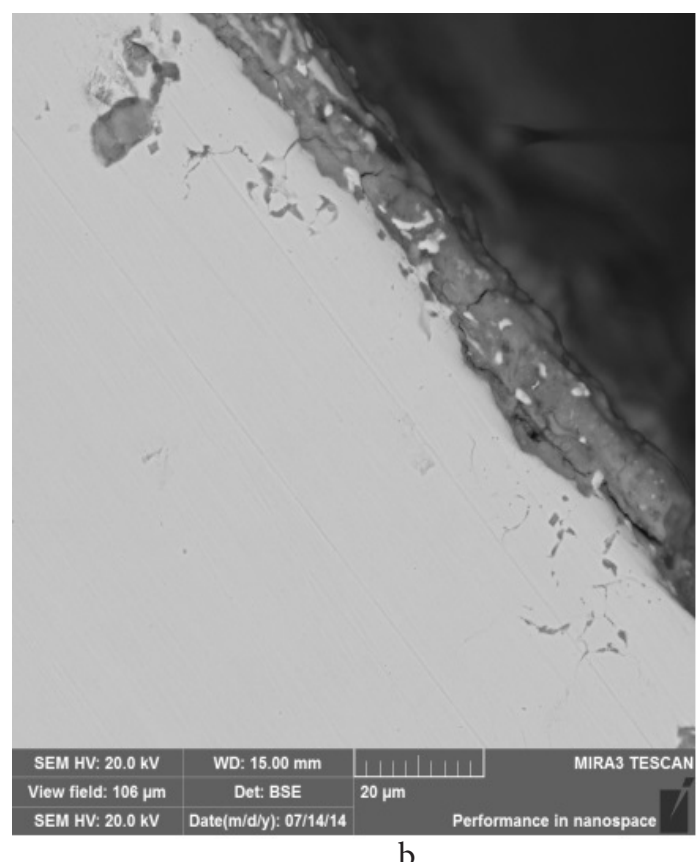

b

Fig. 2. Electron images of the microstructure of the diffusion coatings on steel 45 , produced by complex saturation with boron, chromium and titanium from pastes: (a) saturation from a one-layer paste; (b) saturation from a two-layer paste.

Table 1.

Elemental composition of the coatings produced by simultaneous diffusion saturation with boron, chromium and titanium, based on energy-dispersive analysis data

\begin{tabular}{|c|c|c|c|c|}
\hline \multirow{2}{*}{ Element } & \multicolumn{2}{|c|}{ One-layer paste } & \multicolumn{2}{|c|}{ Two-layer paste } \\
\hline & Wt.\% & Sigma Wt.\% & Wt.\% & Sigma Wt.\% \\
\hline B & 7.64 & 2.32 & 8.40 & 1.69 \\
\hline $\mathrm{O}$ & 4.70 & 0.21 & 38.12 & 0.73 \\
\hline $\mathrm{Si}$ & - & - & 0.73 & 0.04 \\
\hline $\mathrm{Ca}$ & - & - & 0.13 & 0.03 \\
\hline $\mathrm{Ti}$ & 0.19 & 0.04 & 0.79 & 0.04 \\
\hline $\mathrm{Cr}$ & - & - & 0.19 & 0.03 \\
\hline $\mathrm{Mn}$ & 0.71 & 0.07 & 0.69 & 0.05 \\
\hline $\mathrm{Fe}$ & 86.75 & 2.18 & 50.95 & 0.96 \\
\hline
\end{tabular}

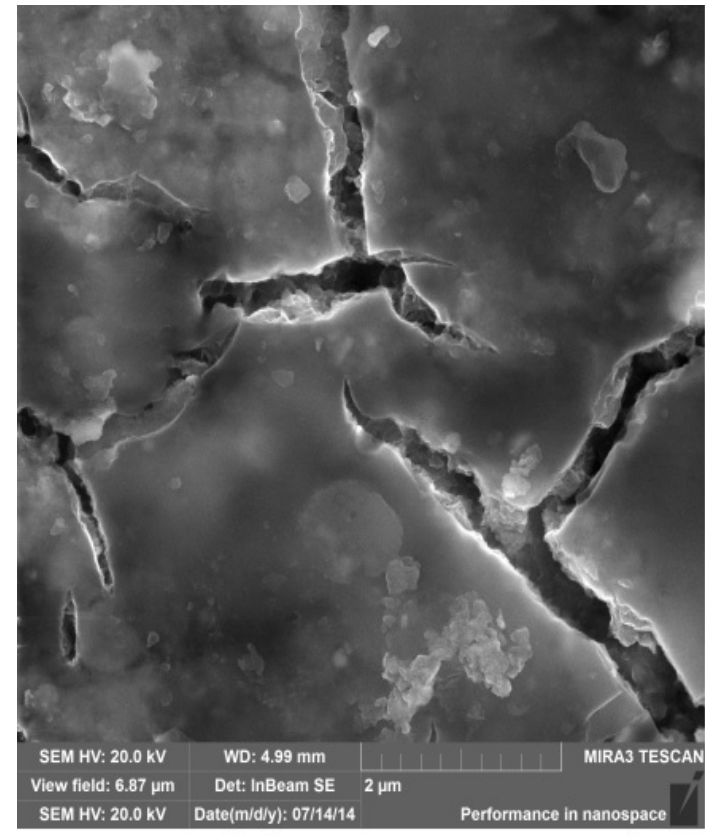

Fig. 3. Image of the surface of the diffusion coating produced by simultaneous saturation with boron, chromium and titanium from a two-layer paste
Table 2.

Elemental composition of hardenable steel 45

\begin{tabular}{|l|l|l|l|l|}
\hline \multirow{2}{*}{ Element } & \multicolumn{2}{|l|}{ One-layer paste } & \multicolumn{2}{l|}{ Two-layer paste } \\
\cline { 2 - 5 } & Wt.\% & $\begin{array}{l}\text { Sigma Wt. } \\
\%\end{array}$ & Wt.\% & $\begin{array}{l}\text { Sigma Wt. } \\
\%\end{array}$ \\
\hline $\mathrm{Si}$ & 0.34 & 0.05 & 0.34 & 0.05 \\
\hline $\mathrm{Mn}$ & 0.79 & 0.07 & 0.78 & 0.07 \\
\hline $\mathrm{Fe}$ & 98.86 & 0.09 & 98.87 & 0.09 \\
\hline
\end{tabular}

The elemental composition of the matrix is given in Table 2, and as can be seen from the table, it corresponds to the basic elemental composition of steel 45 .

\section{Conclusions}

The conducted investigations allow to make the following 
conclusions:

1. The elemental composition and the method of application of saturating paste has a determining influence on the processes of diffusion of active atoms, the conditions of formation and the microstructure of the produced coatings.

2. The elemental composition and the method of application of the saturating paste drastically changes the mechanical characteristics and operating capacity of the hardening coatings on carbon steel 45 .

3. On the basis of the results of the performed work, two pilot batches of knives from steel 45 for grinding polypropylene cases of lead-acid starter batteries were produced. The coating of each batch was obtained from one-layer and two-layer paste, correspondingly. The operating life of the hardened knives from steel 45 was measured from the mass of the processed cases, and was compared between the produced batches and conventional knives made of steel 65G. The operating life of conventional knives from steel 65G was 8-10 tons of processes cases, while the operating life of the knives after boriding from a one-layer paste was 38 tons, and the operating life of the knives hardened from a two-layer paste was about 100 tons of processes cases.

Thus, diffusion saturation from a one-layer paste containing boron and titanium allows to increase the durability of knives from hardened steel 453.8-fold to 4.75-fold, and saturation from a two-layer paste, each layer of which contains either boron or titanium, allows to increase their durability 10 -fold to 12.5 -fold.

This work was supported under RFBR grant \# 13-08-98107, the assignment of the Ministry of Science and Education of the Russian Federation in the priority areas in the development of science and technology (topic \#885) and the grant from the President of the Russian Federation
(Agreement \#14. Z56.14.656-MK).

The electron microscopy studies were carried out at the demo-center "Tescan Ltd".

\section{References}

1. A.M. Guriev, B.D. Ligdenov, S.G. Ivanov, O.A. Vlasova, E.A. Kosheleva, I.A. Garmaeva, M.A. Guriev. Fundamentalnie problemy sovremennogo materialovedenya. 4(1), 30 (2007). (in Russian)

2. A.M. Guriev, S.G. Ivanov. Fundamentalnie problemy sovremennogo materialovedenya. 8(3), 92 (2011). (in Russian)

3. A.M. Guriev, S.G. Ivanov, A.D. Greschilov, S.A. Zemljakov. Obrabotka metallov. (Technologiya oborudovanie instrumenty). 3, 34 (2011). (in Russian)

4. B. D. Ligdenov, A. M. Guriev. Izvestiya vuzov. Fizika. 43 (11), 269 (2000). (in Russian)

5. A.M. Guriev, Yu. P. Kharaev. The Theory and Practice of Producing Cast Tools. (2005) 220 p. (in Russian)

6. A.M. Guriev, S. G. Ivanov, I. A. Garmaeva. Diffusion Coatings on Steels and Alloys. (2013) 221 p. (in Russian)

7. S.G. Ivanov, A.M. Guriev, M.D. Starostenkov, T. G. Ivanova, A. A. Levchenko. Russian Physics Journal. 57(2), 266 (2014). DOI: 10.1007/s11182-014-0234-6

8. Guriev A.M., Kozlov E.V., Krimskikh A.I., Ignatenko L. N., Popova N. A. Izvestiya vuzov. Fizika. 43 (11), 60 (2000). (in Russian)

9. Ivanov S. G., Garmaeva I. A., Androsov A. P., Zobnev V. V., Guriev A.M., Markov V.A. Polzunovskii vestnik. 1(1), 106 (2012). (in Russian)

10. A. M. Guriev, E. V. Kozlov, B. D. Lygdenov, A. M. Kirienko, E. V. Chernyh. Fundamentalnie problemy sovremennogo materialovedenya. 1(2), 54 (2004). (in Russian) 